
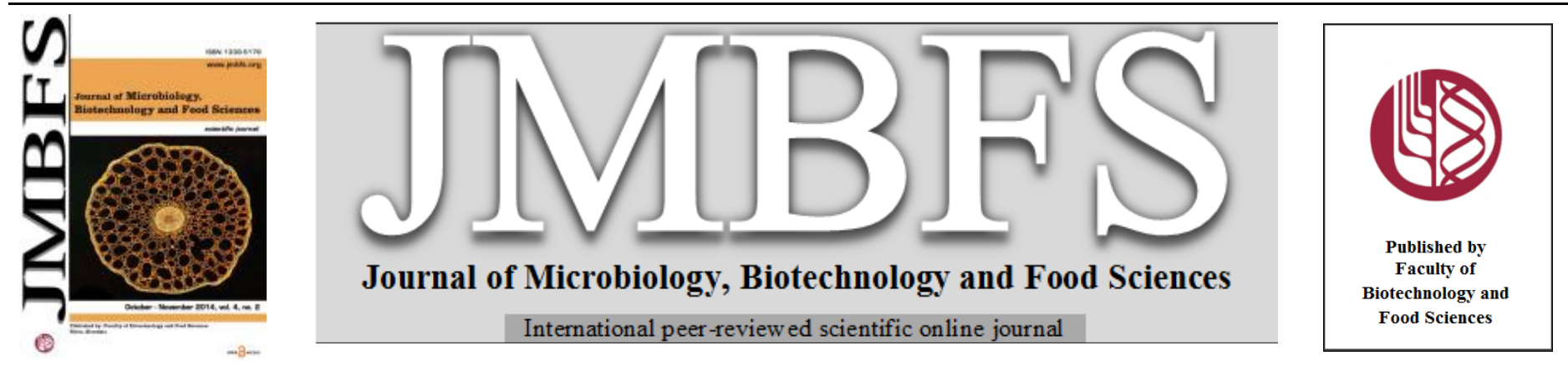

\title{
PRODUCT SAFETY AND COLOR CHARACTERISTICS OF GROUND BEEF PROCESSED FROM BEEF TRIMMINGS TREATED WITH PEROXYACETIC ACID ALONE OR FOLLOWED BY NOVEL ORGANIC ACIDS
}

\author{
Fred Pohlman $^{* 1}$, Palika Dias-Morse ${ }^{1}$, Dilan Pinidiya ${ }^{2}$ \\ Address(es): Fred Pohlman, PhD. \\ ${ }^{1}$ Department of Animal Science, University of Arkansas, Fayetteville AR 72701, USA. \\ ${ }^{2}$ University of Arkansas for Medical Sciences, Little Rock AR 72205, USA.
}

*Corresponding author: fpohlma@uark.edu

doi: $10.15414 / j m b f s .2014 .4 .2 .93-101$

\section{ARTICLE INFO}

Received 24. 10. 2013

Revised 28. 7. 2014

Accepted 28. 7. 2014

Published 1. 10. 2014

Regular article

open $\partial_{\text {AcCESS }}$

\section{ABSTRACT}

The objective of this study was to evaluate the effectiveness of antimicrobial interventions using peroxyacetic acetic acid (PAA) followed by novel organic acids on beef trimmings prior to grinding with conventional spray (CS) or electrostatic spray (ES) on ground beef microbial populations and color. Beef trimmings $(80 / 20 ; 25 \mathrm{~kg}$ ) were inoculated with E. coli $\mathrm{O} 157: \mathrm{H} 7$, non- O157:H7 shiga toxin producing (STEC) E. coli (EC) and Salmonella spp. (SA) at $10^{5} \mathrm{CFU} / \mathrm{g}$. Inoculated trimmings (1.5 kg /treatment/replicate, 2 replicates) were treated with CS application of $0.02 \%$ PAA alone or followed by CS or ES application of 3\% octanoic acid (PO), 3\% pyruvic acid (PP), 3\% malic acid (PM), saturated solution of fumaric acid (PF) or deionized water (W). Findings from this study suggest that PA as a single or multiple chemical hurdle approach with malic, pyruvic, octanoic and fumaric acid on beef trimmings may be effective in reducing E. coli $\mathrm{O} 157: \mathrm{H} 7$ as well as non-STEC serotypes and Salmonella in ground beef up to day 2 of simulated retail display. Results of this study showed that instrumental color properties of ground beef treated with peroxyacetic acid followed by organic acids had little or no difference $(P>0.05)$ compared to the untreated un-inoculated control ground beef samples. The results also indicate that ES application of some organic acids may have similar or greater efficiency in controlling ground beef microbial populations compared to the CS application of the same acid providing a more economical and waste manageable decontamination approach.

Keywords: non-STEC O157:H7, Salmonella spp., meat safety, instrumental color

\section{INTRODUCTION}

The post-harvest processes involved in ground beef production pose a risk to the product microbial safety even under the most cleanest of conditions. In addition, as a rich nutrient matrix, meat provides an excellent media for growth and survival of pathogenic bacteria (Ayemerich et al., 2008; Chen et al., 2012) causing ground beef to often be ources for food-pathogen related outbreaks. Escherichia coli and Salmonella are important food-borne pathogens associated with ground beef and responsible for large numbers of food-borne illness cases in the United States (Edwards and Fung 2006; Mead et al., 1995). Although many effective decontamination interventions have been found and practiced in the beef industry, the concerns associated with the cost, environmental impact, and deleterious impact on ground beef quality attributes, as well as new pathogen emerging give emphasis to the continuous search for new and effective methods of bacterial decontamination methods available to the beef industry. Escherichia coli O157:H7 was responsible for many outbreaks including the incident of 700 cases of enterohemorrhagic illness among people who ate undercooked ground beef in 1993 (Edwards and Fung 2006). On the other hand, non-O157 shiga toxin strains have emerged and especially six serotypes O26, O45, O103, O111, O121, and O145 have been associated with large outbreaks of human disease (Nataro and Kaper, 1998). Further, the U.S. Department of Agriculture Food Safety and Inspection Services highlights the need for further research to reduce Salmonella Typhimurium type 104 (DT104) in meat as it exhibits multi-drug resistance patterns to many antibiotics along with food-borne illness challenges (Hogue et al., 1997). Therefore, applications of decontamination interventions targeting beyond the traditional pathogenic bacteria in the ground beef production line are a crucial step for the beef industry to enhance product microbial safety. Ground beef consumers not only demand for microbial safety but also look for natural ingredients, natural flavor and color, and fewer chemicals present in the product. Organic acids are natural constituents of many foods (Roller, 2003) and are Generally Recognized as Safe (GRAS) antimicrobials (Smulders and Geer 1998). The bactericidal and bacteriostatic efficacy of aqueous organic acids (1$3 \%$ ) in reducing microbial populations in meat carcass processing has been reported (Dickson and Anderson 1992; Edwards and Fung 2006; Hardin et al., 1995; Huffman, 2002; Smulders and Geer 1998). Among organic acids, acetic and lactic acids have been the most widely used in carcas decontamination (Berry and Cutter, 2000; Ransom et al., 2003). Acetic, lactic and propionic acid sprays or washes have been recognized as effective interventions to reduce E. coli and other bacteria on sheep/goat carcasses (Dubal and Patukar 2004; Ramirez et al., 2001). According to Cutter and RiveraBetancourt (2000) spray treatments of $2 \%$ acetic acid or $2 \%$ lactic acid on beef surfaces may effectively reduce E. coli O111:H8 and Salmonella Typhimurium DT 104 to the same extent as E. coli O157:H7 and Salmonella Typhimurium, respectively. In contrast, some research found organic acids were ineffective against inhibiting the growth $E$. coli on beef carcasses (Brakate et al., 1993; Greer and Dilts 1992). The mechanism of action of organic acids on microbial cells has been attributed to cytoplasmic acidification below the microbial growth range along with metabolic inhibition caused by accumulation of undissociated acid molecules (Levine and Fellers 1940). However, organic acid decontamination efficacy largely depends on the type of meat tissue, the type and initial load of microbial contamination (Sofos and Smith 1998) application methods (Edwards and Fung 2006) and acid concentration (Smulders and Geer 1998).

Peroxyacetic acid, a GRAS equilibrium mixture of hydrogen peroxide and peracetic acid, is found to be effective against a variety of pathogenic and spoilage organisms in meat decontamination (Ellebracht et al., 2005; King et al., 2005, Pohlman et al., 2009). Although acetic acid, citric acid, lactic acid have been used in meat decontamination interventions, it would be of value to consider other food grade organic acids which are general metabolic intermediates and have no known toxic health effects at concentrations potential to control or inhibit pathogenic bacteria in ground beef. In recent years, much research specified the importance of antimicrobial interventions on beef trimmings as a pre-grinding treatment in controlling microbial populations in ground beef (Pohlman et al., 2009; 2002a; 2002b; Quilo et al., 2010). In addition, scientific evidence support that multiple hurdles approach may obtain greater reduction of microbial populations than using a single intervention (Pohlman et al., 2009; 2002a; 2002b). The spray application via electrostatic atomization of liquid is an emerging technology that has the potential to be used in beef antimicrobial decontamination interventions as it has the ability to produce very even and uniform antimicrobial liquid coating applications (AbuAli and Barringer 2005). Therefore, the objective of this study was to explore multiple chemical hurdle intervention strategies using peroxyacetic acid followed 
by under-utilized organic acids such as octanoic, pyruvic, malic and fumaric acid on beef trimmings to control pathogenic bacteria in ground beef and its impact on ground beef instrumental color attributes. Further the efficiencies of electrostatic spray and conventional spray application methods were compared to develop cost-conscious intervention approaches targeted at maximum reduction of traditional bacteria as well as the new emerging strains with a negligible impact on quality attributes.

\section{MATERIAL AND METHODS}

\section{Microorganisms}

Escherichia coli $\mathrm{O} 157: \mathrm{H7}$, non-O157:H7 Shiga toxin producing E. coli O26, O103, O111, O121, O45 and O145 (EC), Salmonella Typhimurium DT 104, and S. Newport (SA) were used in this study. All strains were grown in Brian Heart Infusion broth (BHI; DIFCO, Detroit, USA) at $37^{\circ} \mathrm{C}$ and stored at $-80^{\circ} \mathrm{C}$ in $\mathrm{BH}$ with $20 \%$ glycerol (w/v; Amresco, Solon, USA) and maintained in BHI-agar $(1.5 \%)$ slants at $4^{\circ} \mathrm{C}$

\section{Inoculum preparation and growth condition}

One loop of each strain from BHI-agar slants was inoculated into $10 \mathrm{~m}$ individual aliquots of BHI. They were incubated for 18 hours at $37^{\circ} \mathrm{C}$ for 18 hours, non-shaking. Bacterial pellets were obtained by centrifugation $(3500 \mathrm{~g}$ for 20 minutes at $37^{\circ} \mathrm{C}$ ) and were re-suspended in the same volume with buffered peptone water (BPW; Difco, Detroit, USA). Then, equal volumes of each strain was mixed together and further diluted with BPW to achieve a $10^{5} \mathrm{CFU} / \mathrm{ml}$ ninestrain cocktail mixture of EC and SA, and stored at $4{ }^{\circ} \mathrm{C}$ until further use.

\section{Meat Inoculation}

Frozen beef trimmings ( 80 lean/20 fat; $41 \mathrm{~kg}$ ) obtained from Cargill Meat Solutions (Plainview, USA) were thawed, divided equally, and placed in sterile biohazard bags. The cocktail mixture of E.coli (EC) and Salmonella (SA) at $10^{5}$ $\mathrm{CFU} / \mathrm{g}$ was incorporated into the trimming and mixed to acquire an even inoculum distribution on the beef trimmings. Then, the inoculated trimmings were separated into $1.5 \mathrm{~kg}$ batches (replicate/treatment) and left overnight at $4^{\circ} \mathrm{C}$ to allow bacterial attachment (Pohlman et al., 2009; 2002a; 2002b).

\section{Treatment application}

As per manufacturer's recommendations, peroxyacetic acid treatment application was confined to a conventional spray system. The beef trimmings (1.5 $\mathrm{kg} /$ treatment/replicate), prearranged on stainless steel trays, were first treated with conventional spray $(\sim 0.1 \mathrm{ml} / \mathrm{g})$ applications of $0.02 \%$ peroxyacetic acid (PA; FMC, Philadelphia, USA) as a single intervention or followed by conventional (CS) or electrostatic spray (ES; Electrostatic Spraying Systems, Watkinsville, USA) applications $(\sim 0.06 \mathrm{ml} / \mathrm{g})$ of $3 \%$ malic acid (PM; Sigma Aldrich, St. Louis USA), 3\% pyruvic acid (PP; Sigma Aldrich, St. Louis, USA), 3\% octonoic acid (PO; Sigma Aldrich, St. Louis, USA), and a saturated solution of fumaric acid (PF; Sigma Aldrich, St. Louis, USA). The PA- treated samples were allowed to drip for $3 \mathrm{~min}$ prior to and after assigned second antimicrobial applications (2 replicates / treatment). Inoculated beef trimmings were also treated with the conventional and electrostatic spray applications of de-ionized water (W) at the same rates used in antimicrobial applications and allowed to drip for $3 \mathrm{~min}$. Each treatment was applied two times. Untreated inoculated samples were retained as a control $(\mathrm{CON})$.

\section{Meat processing}

Following treatment application, all treated and untreated inoculated $(\mathrm{CON})$ beef trimmings were ground (American Eagle, $32 \mathrm{~cm}$ chopper plate) twice and $200 \mathrm{~g}$ of individual samples were placed on plastic foam trays and over wrapped with polyvinyl chloride film $\left(\mathrm{O}_{2}\right.$ transmission rate $=14,000 \mathrm{cc} / \mathrm{mm} 2 / 24 \mathrm{~h} / 1 \mathrm{~atm} ; \mathrm{Koch}$ Kansas City, USA). The ground beef packages were displayed under retail condition $\left(4^{\circ} \mathrm{C} ; 1,630 \mathrm{~lx}\right.$ of deluxe warm white fluorescent lighting; Phillips Inc.,
Somerset, USA) and sampled on day $0,1,2,3$, and 7 day of display for microbial analysis and CIE L*, a* and $\mathrm{b}^{*}$ and reflectance measurements. The $\mathrm{pH}$ of ground beef was monitored (homogenized mixture of $1.8 \mathrm{~g}$ of ground beef and $18 \mathrm{ml}$ of distilled water; (Quilo et al., 2009) at $27^{\circ} \mathrm{C}$, using a Orion $\mathrm{pH}$ meter with Orion Ross ultra electrode (Fisher Scientific, Houston, USA) at day 0, 1, 2, 3, and 7 days of display (Pohlman et al., 2009; 2002a; 2002b).

\section{Microbial enumeration}

On the appropriate day of display, a sample of $25 \mathrm{~g}$ was aseptically removed from each ground beef sample and placed in sterile whirlpack bags (Nasco, Ft Atkinson, USA) separately. These samples were incorporated with $225 \mathrm{ml}$ of $0.1 \%$ buffered peptone water and homogenized for 2 minutes using a stomacher (Seward, London, UK). Subsequently, serial 10-fold dilutions were made and duplicate spread plated (SA counts on Salmonella shigella agar (DIFCO, Detroit, USA), aerobic plate count (APC), and E. coli (EC) / coliform (CO) counts on Petrifilm $^{\circledR}$ (3M, St. Paul, USA). The EC, APC and ST counts were read after $48 \mathrm{~h}$ incubation at $37{ }^{\circ} \mathrm{C}$ (VWR Scientific, West Chester, USA) whereas coliform plates were read after 24 h (Pohlman et al., 2009; 2002a; 2002b).

\section{Instrumental color}

Instrumental color was measured ( $\mathrm{n}=3 /$ treatment) using a Hunter-Lab MiniScan XE Spectrocolorimeter (Reston, USA). Prior to measurements of samples, the Spectrocolorimeter was standardized using a white tile, black tile and working standards (Pohlman et al., 2002a; 2002b). The ground beef samples were evaluated for CIE $L^{*}$ (lightness), $a^{*}$ (redness), and $b^{*}$ (yellowness) using Illuminant $\mathrm{A} / 10^{\circ}$ observer. Additionally, a reflectance measurement in the 580 to $630 \mathrm{~nm}$ visible spectrum was acquired. The hue angle, saturation index and reflectance ratio were calculated as $\left(\arctan (b * / a *),\left(\left(a^{* 2}+b^{* 2}\right)\right)^{0.5}\right.$, and $(630 / 580$ $\mathrm{nm}$ ), respectively (Quilo et al., 2009a; 2009b).

\section{Statistical analysis}

The experimental design was a randomized complete block. The experimenta design included antimicrobial treatments, application methods and 5 display days $(0,1,2,3$ and 7$)$ as factors in the randomized complete block design. Treatment were blocked by replicate and then analyzed for the main effects of antimicrobia treatment, day of display and treatment by day interactions. Least squares means for significant main effects were identified using the LSMEANS and separated using the PDIFF option of SAS (version 9.2, SAS Institute Inc., Cary, USA).

\section{RESULTS AND DISCUSSION}

\section{Ground beef microbial populations}

\section{Coliform}

A significant reduction $(P<0.05)$ in coliform counts in all treated-ground beef was noticeable compared to the inoculated un-treated control on day 0 (Table 1.1). However, ground beef processed from PA, W and PM treatments by CS application, PM, PP, and PF treatments by ES application achieved over 1 log reduction $(P<0.05)$ of ground beef coliforms (CO) compared to CON Considering all the treatments and application methods, PM $(1.8 \mathrm{log})$ and PP $(1.75 \mathrm{log})$ by $\mathrm{CS}$ method were more efficient in $\mathrm{CO} \log$ reduction $(P<0.05)$ for day 1 of display. On the other hand, PA, W and PF by ES, PP by CS treatments exceeded $(P<0.05)$ the other treatments in controlling CO counts with more than $1 \log$ reduction on day 2 of display. None of the treatments showed significant $\mathrm{CO}$ reductions $(P>0.05)$ on day 7 of display. The PP treatment applied through ES system outperformed the CS application in controlling ground beef $\mathrm{CO}$ population on day 0 of display. Nevertheless, there was no significant difference $(P>0.05)$ between CS vs. ES methods of $\mathrm{W}, \mathrm{PM}, \mathrm{PO}$ and PF treatments in ground beef $\mathrm{CO}$ reduction on day 0 of display. Therefore, ES application of these antimicrobials was able to achieve similar CO reduction as CS, but with much less antimicrobial usage and over-spray wastage. 
Table 1.1 Effects of antimicrobial treatment, application method and day of display against coliform population in ground beef during simulated retail display storage at $4^{\circ} \mathrm{C}$

\begin{tabular}{|c|c|c|c|c|c|c|}
\hline \multirow{2}{*}{ *Treatment } & \multirow{2}{*}{ **Application Method } & \multicolumn{5}{|c|}{ Coliform count (log CFU/g) } \\
\hline & & Day 0 & Day 1 & Day 2 & Day 3 & Day 7 \\
\hline $\mathrm{CON}$ & - & $5.17^{\mathrm{a}}$ & $5.51^{\mathrm{a}}$ & $5.74^{\mathrm{a}}$ & $6.02^{\mathrm{b}}$ & 7.09 \\
\hline PA & $\mathrm{CS}$ & $3.74^{\mathrm{d}}$ & $4.62^{\mathrm{bc}}$ & $4.56^{\mathrm{d}}$ & $5.98^{\mathrm{b}}$ & 6.79 \\
\hline W & $\mathrm{CS}$ & $4.12^{\mathrm{bc}}$ & $5.67^{\mathrm{a}}$ & $5.73^{\mathrm{a}}$ & $6.27^{\mathrm{a}}$ & 7.09 \\
\hline W & ES & $4.39^{\mathrm{b}}$ & $5.47^{\mathrm{a}}$ & $4.56^{\mathrm{d}}$ & $5.98^{\mathrm{b}}$ & 6.86 \\
\hline PM & $\mathrm{CS}$ & $3.94^{\mathrm{cd}}$ & $3.71^{\mathrm{d}}$ & $4.91^{\mathrm{cd}}$ & $5.26^{\mathrm{f}}$ & 6.89 \\
\hline PM & ES & $4.16^{\mathrm{bc}}$ & $4.97^{\mathrm{b}}$ & $5.42^{\mathrm{ab}}$ & $6.00^{\mathrm{b}}$ & 6.60 \\
\hline PP & $\mathrm{CS}$ & $4.22^{\mathrm{cb}}$ & $3.76^{\mathrm{d}}$ & $4.03^{\mathrm{e}}$ & $5.79^{d}$ & 6.68 \\
\hline PP & ES & $3.73^{\mathrm{d}}$ & $4.40^{\mathrm{c}}$ & $4.84^{\mathrm{cd}}$ & $5.45^{\mathrm{e}}$ & 6.60 \\
\hline $\mathrm{PO}$ & $\mathrm{CS}$ & $4.30^{\mathrm{b}}$ & $4.53^{\mathrm{bc}}$ & $5.61^{\mathrm{a}}$ & $5.56^{\mathrm{e}}$ & 6.78 \\
\hline $\mathrm{PO}$ & ES & $4.33^{\mathrm{b}}$ & $4.49^{c}$ & $5.08^{\mathrm{bc}}$ & $5.54^{\mathrm{e}}$ & 6.51 \\
\hline $\mathrm{PF}$ & $\mathrm{CS}$ & $4.30^{\mathrm{b}}$ & $4.55^{\mathrm{bc}}$ & $5.41^{\mathrm{ab}}$ & $5.45^{\mathrm{e}}$ & 6.74 \\
\hline \multirow[t]{2}{*}{$\mathrm{PF}$} & ES & $4.15^{\mathrm{bc}}$ & $4.65^{\mathrm{bc}}$ & $4.87^{\mathrm{cd}}$ & $5.48^{\mathrm{e}}$ & 6.95 \\
\hline & Standard error & \pm 0.06 & \pm 0.09 & \pm 0.07 & \pm 0.02 & \pm 0.12 \\
\hline
\end{tabular}

Coliform count (log Colony Forming Units/g) reported as least squares means along with \pm standard error.

${ }^{\mathrm{a}-\mathrm{d}}$ Least squares means within a column with different superscripts differed significantly $(P<0.05)$.

"Treatments: $\mathrm{CON}=$ untreated inoculated control, $\mathrm{PA}=0.02 \%$ peroxyacetic acid; $\mathrm{W}=$ deionized water, $\mathrm{PM}=0.02 \%$ peroxyacetic acid followed by $3 \%$ malic acid, $\mathrm{PP}=0.02 \%$ peroxyacetic acid followed by $3 \%$ pyruvic acid, $\mathrm{PO}=0.02 \%$ peroxyacetic acid followed by $3 \%$ octanoic acid, $\mathrm{PF}=$ $0.02 \%$ peroxyacetic acid followed by saturated solution of fumaric acid.

**Application methods: $\mathrm{CS}=$ conventional spray application, $\mathrm{ES}=$ electrostatic spray application.

\section{Escherichia coli}

Ground beef processed from PA, W, and PP, treatments applied through CS method achieved $1 \log$ reduction or more in Escherichia coli (EC) on day 0 of display (Table 1.2). The PM, PP treatments applied through CS outperformed all the other treatments and achieved the lowest $(P<0.05)$ EC count compared to the control on day 1 of display with up to or above $1.9 \log$ reduction. However, $\mathrm{PO}$ and PF treatments by both application methods together with PM and PP applied through the ES system also possessed significantly lower $(P<0.05) \mathrm{EC}$ counts compared to the control with 1 or more $\log$ reduction on day 1 of display. While PA along with ES application of PP, PO, and PF showed $>1 \log$ reduction, CS application of PM and PP treatments accounted for $>2 \log$ reduction in EC counts on day 2 of display. The CS and ES application methods showed no difference $(\mathrm{P}>0.05)$ in lowering EC counts for PP, PO, or PF treatments on day 3 of display. On the contrary, PP and PM treatments were more efficient $(P<0.05)$ with the CS application method compared to the ES method in lowering ground beef EC populations on day 1 and 2 of display, respectively.

Table 1.2 Effects of antimicrobial treatment, application method and day of display against Escherichia coli in ground beef during simulated retail display storage at $4^{\circ} \mathrm{C}$

\begin{tabular}{|c|c|c|c|c|c|c|c|}
\hline \multirow{2}{*}{ *Treatment } & \multirow{2}{*}{ **Application Method } & & \multicolumn{5}{|c|}{ Escherichia coli count $(\log \mathrm{CFU} / \mathrm{g})$} \\
\hline & & & Day 0 & Day 1 & Day 2 & Day 3 & Day 7 \\
\hline $\mathrm{CON}$ & - & & $5.22^{\mathrm{a}}$ & $5.77^{\mathrm{ab}}$ & $6.29^{\mathrm{a}}$ & $6.30^{\mathrm{a}}$ & $7.33^{\mathrm{a}}$ \\
\hline PA & $\mathrm{CS}$ & & $3.77^{\mathrm{f}}$ & $4.88^{\mathrm{c}}$ & $4.96^{\mathrm{f}}$ & $6.19^{\mathrm{ab}}$ & $7.19^{\mathrm{b}}$ \\
\hline $\mathrm{W}$ & CS & & $4.22^{\mathrm{d}}$ & $5.90^{\mathrm{a}}$ & $6.02^{\mathrm{b}}$ & $6.31^{\mathrm{a}}$ & $7.28^{\mathrm{a}}$ \\
\hline $\mathrm{W}$ & ES & & $4.43^{\mathrm{c}}$ & $5.71^{\mathrm{b}}$ & $5.73^{\mathrm{c}}$ & $6.28^{\mathrm{ab}}$ & $7.13^{\mathrm{c}}$ \\
\hline PM & $\mathrm{CS}$ & & $4.35^{\mathrm{cd}}$ & $3.82^{\mathrm{e}}$ & $4.09^{\mathrm{g}}$ & $5.49^{\mathrm{d}}$ & $7.13^{\mathrm{c}}$ \\
\hline PM & ES & & $4.39^{\mathrm{cd}}$ & $4.68^{\mathrm{d}}$ & $5.44^{\mathrm{d}}$ & $6.27^{\mathrm{ab}}$ & $6.96^{\mathrm{d}}$ \\
\hline PP & CS & & $3.99^{\mathrm{e}}$ & $3.84^{\mathrm{e}}$ & $4.03^{\mathrm{g}}$ & $5.60^{\mathrm{cd}}$ & $7.01^{\mathrm{d}}$ \\
\hline $\mathrm{PP}$ & ES & & $4.81^{\mathrm{b}}$ & $4.62^{\mathrm{d}}$ & $4.95^{\mathrm{f}}$ & $5.77^{\mathrm{cd}}$ & $7.21^{\mathrm{b}}$ \\
\hline $\mathrm{PO}$ & $\mathrm{CS}$ & & $4.47^{\mathrm{c}}$ & $4.72^{\mathrm{d}}$ & $5.95^{\mathrm{b}}$ & $5.80^{\mathrm{cd}}$ & $6.97^{\mathrm{d}}$ \\
\hline $\mathrm{PO}$ & ES & & $4.45^{\mathrm{c}}$ & $4.67^{\mathrm{d}}$ & $5.14^{\mathrm{e}}$ & $5.70^{\text {cd }}$ & $7.12^{\mathrm{c}}$ \\
\hline PF & $\mathrm{CS}$ & & $4.32^{\mathrm{cd}}$ & $4.62^{\mathrm{d}}$ & $5.81^{\mathrm{c}}$ & $5.92^{\mathrm{bc}}$ & $7.12^{\circ}$ \\
\hline PF & ES & & $4.29^{\mathrm{cd}}$ & $4.77^{\mathrm{cd}}$ & $5.06^{\mathrm{e}}$ & $5.76^{\mathrm{cd}}$ & $7.11^{\circ}$ \\
\hline & & Standard error & \pm 0.04 & \pm 0.03 & \pm 0.02 & \pm 0.08 & \pm 0.01 \\
\hline
\end{tabular}

Escherichia coli count (log Colony Forming Units/g) reported as least squares means along with \pm standard error.

${ }^{\mathrm{a}-\mathrm{g}}$ Least squares means within a column with different superscripts differed significantly $(P<0.05)$.

"Treatments: $\mathrm{CON}=$ untreated inoculated control, $\mathrm{PA}=0.02 \%$ peroxyacetic acid; $\mathrm{W}=$ deionized water, $\mathrm{PM}=0.02 \%$ peroxyacetic acid followed by $3 \%$ malic acid, $\mathrm{PP}=0.02 \%$ peroxyacetic acid followed by $3 \%$ pyruvic acid, $\mathrm{PO}=0.02 \%$ peroxyacetic acid followed by $3 \%$ octanoic acid, $\mathrm{PF}=$ $0.02 \%$ peroxyacetic acid followed by saturated solution of fumaric acid.

**Application methods: CS = conventional spray application, $\mathrm{ES}=$ electrostatic spray application.

\section{Aerobic plate counts}

The PA and PO-treated ground beef through CS application lead by CS application of PP, showed the lowest $(P<0.05)$ aerobic plate count (APC) on day 0 of display (Table 1.3). On day 1 of display CS application of PM treatments along with PP and PF treatments applied by both methods obtained over $2 \log$ reduction of ground beef APC. The PF treated ground beef through CS application reported the lowest $(P<0.05)$ APC with $2.05 \log$ reduction on day 2 of display. Although ground beef from PP treated ground beef by ES had less performance $(P<0.05)$ compared to CS, it accounted for more than $1 \log$ reduction of APC on day 2 of display. Further, the PP by ES treatment was able to maintain a $1.02 \log$ reduction of APC on day 3 of display. Both ES and CS treatment application methods of PO and PF treatments showed a similar $(P>$ $0.05)$ efficiency in controlling ground beef APC on day 1 of display. All treatments regardless of application method were effective for reducing $(P<$ $0.05)$ APC by day 7 of display. 
Table 1.3 Effects of antimicrobial treatment, application method and day of display against total aerobic plate bacteria in ground beef during simulated retail display storage at $4^{\circ} \mathrm{C}$

\begin{tabular}{|c|c|c|c|c|c|c|}
\hline \multirow{2}{*}{$*$ Treatment } & \multirow{2}{*}{ **Application Method } & \multicolumn{5}{|c|}{ Aerobic plate count (log CFU/g) } \\
\hline & & Day 0 & Day 1 & Day 2 & Day 3 & Day 7 \\
\hline $\mathrm{CON}$ & - & $5.52^{\mathrm{b}}$ & $6.27^{\mathrm{a}}$ & $6.33^{\mathrm{a}}$ & $6.59^{\mathrm{abc}}$ & $7.89^{\mathrm{a}}$ \\
\hline PA & CS & $4.57^{\mathrm{f}}$ & $4.95^{\mathrm{d}}$ & $6.26^{\mathrm{ab}}$ & $6.34^{\text {bcd }}$ & $7.56^{\mathrm{b}}$ \\
\hline $\mathrm{W}$ & CS & $5.04^{\mathrm{d}}$ & $6.19^{\mathrm{a}}$ & $6.08^{\mathrm{abc}}$ & $6.80^{\mathrm{a}}$ & $7.52^{\mathrm{bc}}$ \\
\hline W & ES & $5.64^{\mathrm{a}}$ & $5.66^{\mathrm{b}}$ & $6.21^{\mathrm{ab}}$ & $6.68^{\mathrm{ab}}$ & $7.24^{\mathrm{d}}$ \\
\hline PM & CS & $5.48^{\mathrm{b}}$ & $4.09^{\mathrm{ef}}$ & $5.89^{\text {bcde }}$ & $5.67^{\mathrm{hg}}$ & $7.32^{\mathrm{cd}}$ \\
\hline PM & ES & $5.47^{\mathrm{b}}$ & $4.92^{\mathrm{d}}$ & $5.68^{\mathrm{de}}$ & $6.33^{\text {bcd }}$ & $7.36^{\mathrm{bcd}}$ \\
\hline PP & CS & $4.36^{\mathrm{g}}$ & $4.00^{\mathrm{f}}$ & $4.28^{\mathrm{g}}$ & $5.94^{\text {efg }}$ & $7.54^{\mathrm{b}}$ \\
\hline PP & ES & $5.11^{\mathrm{d}}$ & $4.14^{\mathrm{e}}$ & $5.14^{\mathrm{f}}$ & $6.12^{\text {def }}$ & $7.51^{\mathrm{bc}}$ \\
\hline PO & CS & $4.60^{\mathrm{f}}$ & $5.04^{\mathrm{c}}$ & $5.98^{\mathrm{abcd}}$ & $6.28^{\text {cde }}$ & $7.38^{\mathrm{bcd}}$ \\
\hline $\mathrm{PO}$ & ES & $4.77^{\mathrm{e}}$ & $5.05^{\mathrm{c}}$ & $5.59^{\mathrm{e}}$ & $5.94^{\mathrm{efg}}$ & $7.30^{\mathrm{d}}$ \\
\hline PF & CS & $5.30^{\mathrm{c}}$ & $4.11^{\mathrm{e}}$ & $5.98^{\mathrm{abcd}}$ & $5.86^{\mathrm{fgh}}$ & $7.50^{\mathrm{bc}}$ \\
\hline PF & ES & $4.79^{\mathrm{e}}$ & $4.06^{\mathrm{ef}}$ & $5.74^{\text {cde }}$ & $5.57^{\mathrm{h}}$ & $7.37^{\mathrm{bcd}}$ \\
\hline & & \pm 0.02 & \pm 0.02 & \pm 0.07 & \pm 0.07 & \pm 0.04 \\
\hline
\end{tabular}

Total aerobic bacterial count (log Colony Forming Units/g) reported as least squares means along with \pm standard error.

${ }^{\text {a-h }}$ Least squares means within a column with different superscripts differed significantly $(P<0.05)$.

"Treatments: $\mathrm{CON}=$ untreated inoculated control, $\mathrm{PA}=0.02 \%$ peroxyacetic acid; $\mathrm{W}=$ deionized water, $\mathrm{PM}=0.02 \%$ peroxyacetic acid followed by $3 \%$ malic acid, $\mathrm{PP}=0.02 \%$ peroxyacetic acid followed by $3 \%$ pyruvic acid, $\mathrm{PO}=0.02 \%$ peroxyacetic acid followed by $3 \%$ octanoic acid, $\mathrm{PF}=$ $0.02 \%$ peroxyacetic acid followed by saturated solution of fumaric acid.

**Application methods: CS = conventional spray application, ES = electrostatic spray application.

\section{Salmonella}

Ground beef processed from PA, ES application of PM, PP, and PF along with CS application of PP and PO reduced $(P<0.05)$ Salmonella $(\mathrm{ST})$ population with over $1 \log$ reduction on day 0 of display (Table 1.4). These treatments together with PO treatment through ES and CS application of PM and PF had significantly lower $(P<0.05)$ Salmonella populations compared to CON on days 1 and 2 of display. The CS application of PP had the lowest $(P<0.05)$ ground beef Salmonella count on day 7 of display. The ES applications of PM and PP had a greater $(P<0.05)$ ST count reductions compared to CS applications of same organic acids on day 0 of display. However, PM, PP, and PF treatments applied by both methods showed similar $(P>0.05)$ ST reduction on day 1 of display. By day 1 of display, all treatments and application methods, with the exception of $\mathrm{W}$ applied by CS, were effective $(P<0.05)$ for reducing Salmonella counts.

Table 1.4 Effects of antimicrobial treatment, application method and day of display against Salmonella Typhimurium in ground beef during simulated retail display storage at $4^{\circ} \mathrm{C}$

\begin{tabular}{|c|c|c|c|c|c|c|c|}
\hline \multirow{2}{*}{ *Treatment } & \multirow{2}{*}{ **Application Method } & & \multicolumn{5}{|c|}{ Salmonella count (log CFU/g) } \\
\hline & & & Day 0 & Day 1 & Day 2 & Day 3 & Day 7 \\
\hline $\mathrm{CON}$ & - & & $5.06^{\mathrm{a}}$ & $5.58^{\mathrm{a}}$ & $6.24^{\mathrm{a}}$ & $6.30^{\mathrm{a}}$ & $7.54^{\mathrm{a}}$ \\
\hline PA & CS & & $3.30^{\mathrm{f}}$ & $3.88^{\text {ed }}$ & $5.01^{\mathrm{d}}$ & $6.19^{\mathrm{ab}}$ & $6.63^{\mathrm{d}}$ \\
\hline W & $\mathrm{CS}$ & & $4.11^{\mathrm{bc}}$ & $5.46^{\mathrm{a}}$ & $6.27^{\mathrm{a}}$ & $6.31^{\mathrm{a}}$ & $6.59^{\mathrm{d}}$ \\
\hline $\mathrm{W}$ & ES & & $4.24^{\mathrm{bc}}$ & $4.31^{\mathrm{cd}}$ & $5.45^{\mathrm{b}}$ & $6.28^{\mathrm{ab}}$ & $6.84^{\mathrm{cd}}$ \\
\hline PM & CS & & $4.14^{\mathrm{bc}}$ & $3.52^{\mathrm{fg}}$ & $3.86^{\mathrm{i}}$ & $5.49^{\mathrm{d}}$ & $6.80^{\text {cd }}$ \\
\hline PM & ES & & $3.39^{\mathrm{ef}}$ & $3.67^{\mathrm{fg}}$ & $4.82^{\mathrm{e}}$ & $6.27^{\mathrm{ab}}$ & $7.27^{\mathrm{ab}}$ \\
\hline PP & CS & & $3.88^{\mathrm{cd}}$ & $3.44^{\mathrm{g}}$ & $3.72^{\mathrm{j}}$ & $5.60^{\mathrm{cd}}$ & $6.12^{\mathrm{e}}$ \\
\hline PP & ES & & $3.15^{\mathrm{f}}$ & $3.56^{\mathrm{fg}}$ & $4.17^{\mathrm{g}}$ & $5.77^{\mathrm{cd}}$ & $7.14^{\mathrm{bc}}$ \\
\hline $\mathrm{PO}$ & CS & & $3.71^{\mathrm{de}}$ & $3.71^{\mathrm{fg}}$ & $4.22^{\mathrm{g}}$ & $5.80^{\mathrm{cd}}$ & $7.02^{\mathrm{bc}}$ \\
\hline $\mathrm{PO}$ & ES & & $4.27^{\mathrm{b}}$ & $4.08^{\text {ed }}$ & $4.05^{\mathrm{h}}$ & $5.70^{\text {cd }}$ & $7.07^{\mathrm{bc}}$ \\
\hline PF & CS & & $4.09^{\mathrm{bc}}$ & $4.68^{\mathrm{b}}$ & $5.26^{\mathrm{c}}$ & $5.92^{\mathrm{bc}}$ & $7.01^{\mathrm{bc}}$ \\
\hline \multirow[t]{2}{*}{$\mathrm{PF}$} & ES & & $3.91^{\mathrm{bcd}}$ & $4.62^{\mathrm{bc}}$ & $4.64^{\mathrm{f}}$ & $5.76^{\mathrm{cd}}$ & $7.14^{\mathrm{bc}}$ \\
\hline & & Standard error & \pm 0.08 & \pm 0.07 & \pm 0.02 & \pm 0.07 & \pm 0.07 \\
\hline
\end{tabular}

Total Salmonella species (log Colony Forming Units/g) reported as least squares means along with \pm standard error

${ }^{a-h}$ Least squares means within a column with different superscripts differed significantly $(P<0.05)$.

"Treatments: $\mathrm{CON}=$ untreated inoculated control, $\mathrm{PA}=0.02 \%$ peroxyacetic acid; $\mathrm{W}=$ deionized water, $\mathrm{PM}=0.02 \%$ peroxyacetic acid followed by $3 \%$ malic acid, $\mathrm{PP}=0.02 \%$ peroxyacetic acid followed by $3 \%$ pyruvic acid, $\mathrm{PO}=0.02 \%$ peroxyacetic acid followed by $3 \%$ octanoic acid, $\mathrm{PF}=$ $0.02 \%$ peroxyacetic acid followed by saturated solution of fumaric acid.

**Application methods: $\mathrm{CS}=$ conventional spray application, $\mathrm{ES}=$ electrostatic spray application.

\section{Ground beef instrumental color properties}

The PA, W, PM and PF treated ground beef had similar lightness $\left(\mathrm{L}^{*}\right)(P>0.05)$ to $\mathrm{CON}$ on days $0,1,3$ and 7 of display regardless of the treatment application method (Table 2.1). Ground beef from beef trimmings treated with PP by both application methods had similar lightness to the CON on days 0,3 and 7 of display. In contrast, the ground beef processed from PO treatment applied by the ES method was significantly $(P<0.05)$ lighter compared to the control through the entire duration of display. 
Table 2.1 Effects of antimicrobial treatment, application method and day of display on ground beef lightness ( $\mathrm{L}^{*}$ ) during simulated retail display storage at $4^{\circ} \mathrm{C}$

\begin{tabular}{|c|c|c|c|c|c|c|c|}
\hline \multirow{2}{*}{$*$ Treatment } & \multirow{2}{*}{ **Application Method } & & \multicolumn{5}{|c|}{ Lightness (L*) } \\
\hline & & & Day 0 & Day 1 & Day 2 & Day 3 & Day 7 \\
\hline $\mathrm{CON}$ & - & & $51.08^{\mathrm{c}}$ & $50.45^{\mathrm{c}}$ & $48.59^{\mathrm{c}}$ & $46.98^{b}$ & $46.42^{b}$ \\
\hline PA & $\mathrm{CS}$ & & $54.45^{\mathrm{abc}}$ & $54.84^{\mathrm{abc}}$ & $53.74^{\mathrm{abc}}$ & $51.11^{\mathrm{ab}}$ & $50.25^{\mathrm{ab}}$ \\
\hline W & $\mathrm{CS}$ & & $53.61^{\mathrm{abc}}$ & $53.83^{\mathrm{bc}}$ & $51.92^{\mathrm{abc}}$ & $51.31^{\mathrm{ab}}$ & $49.86^{\mathrm{ab}}$ \\
\hline W & ES & & $53.53^{\mathrm{abc}}$ & $52.48^{\mathrm{bc}}$ & $49.46^{\mathrm{bc}}$ & $48.03^{\mathrm{b}}$ & $47.05^{\mathrm{ab}}$ \\
\hline PM & $\mathrm{CS}$ & & $54.55^{\mathrm{abc}}$ & $55.86^{\mathrm{abc}}$ & $55.23^{\mathrm{ab}}$ & $52.49^{\mathrm{ab}}$ & $50.43^{\mathrm{ab}}$ \\
\hline PM & ES & & $54.78^{\mathrm{abc}}$ & $55.14^{\mathrm{abc}}$ & $50.91^{\mathrm{abc}}$ & $50.44^{\mathrm{ab}}$ & $51.13^{\mathrm{ab}}$ \\
\hline PP & $\mathrm{CS}$ & & $55.59^{\mathrm{abc}}$ & $56.36^{\mathrm{ab}}$ & $56.38^{\mathrm{a}}$ & $53.84^{\mathrm{ab}}$ & $51.24^{\mathrm{ab}}$ \\
\hline PP & ES & & $53.79^{\mathrm{abc}}$ & $54.56^{\mathrm{abc}}$ & $51.05^{\mathrm{abc}}$ & $49.65^{\mathrm{ab}}$ & $48.30^{\mathrm{ab}}$ \\
\hline $\mathrm{PO}$ & $\mathrm{CS}$ & & $56.73^{\mathrm{ab}}$ & $56.53^{\mathrm{ab}}$ & $54.11^{\mathrm{abc}}$ & $53.07^{\mathrm{ab}}$ & $51.49^{\mathrm{ab}}$ \\
\hline $\mathrm{PO}$ & $\mathrm{ES}$ & & $58.45^{\mathrm{a}}$ & $59.56^{\mathrm{a}}$ & $56.19^{\mathrm{a}}$ & $56.34^{\mathrm{a}}$ & $54.28^{\mathrm{a}}$ \\
\hline $\mathrm{PF}$ & $\mathrm{CS}$ & & $52.78^{\mathrm{bc}}$ & $53.48^{c}$ & $50.95^{\mathrm{abc}}$ & $48.78^{\mathrm{ab}}$ & $47.60^{\mathrm{ab}}$ \\
\hline \multirow[t]{2}{*}{$\mathrm{PF}$} & ES & & $52.68^{\mathrm{bc}}$ & $54.35^{\text {abc }}$ & $51.85^{\mathrm{abc}}$ & $48.94^{\mathrm{ab}}$ & $48.06^{\mathrm{ab}}$ \\
\hline & & Standard error & \pm 0.97 & \pm 0.98 & \pm 1.17 & \pm 1.43 & \pm 1.39 \\
\hline
\end{tabular}

Lightness ( $\mathrm{L}^{*}$ ) reported as least squares means along with \pm standard error,

${ }^{\mathrm{a}-\mathrm{c}}$ Least squares means within a column with different superscripts differed significantly $(P<0.05)$.

"Treatments: $\mathrm{CON}=$ untreated inoculated control, $\mathrm{PA}=0.02 \%$ peroxyacetic acid; $\mathrm{W}=$ deionized water, $\mathrm{PM}=0.02 \%$ peroxyacetic acid followed by $3 \%$ malic acid, $\mathrm{PP}=0.02 \%$ peroxyacetic acid followed by $3 \%$ pyruvic acid, $\mathrm{PO}=0.02 \%$ peroxyacetic acid followed by $3 \%$ octanoic acid, $\mathrm{PF}=$ $0.02 \%$ peroxyacetic acid followed by saturated solution of fumaric acid.

**Application methods: CS = conventional spray application, ES = electrostatic spray application.

Excluding ES applications of PP and PF treatments, all the treatments, despite of application method, had similar $(\mathrm{P}>0.05)$ redness $\left(\mathrm{a}^{*}\right)$ to $\mathrm{CON}$ on day 0 of display (Table 2.2). However, ground beef from PP and PF applied with ES showed higher redness $(P<0.05)$ values compared to the control on day 0 of display. All the treatments applied through CS and ES methods, except ES application of malic acid on day 7 of display, maintained a similar redness to control during days 1 through 7 of display. The treatment and application method did not show an effect on ground beef yellowness on days 0 through 7 of display with all treatments being similar in yellowness to the control and each other (Table 2.3).

Table 2.2 Effects of antimicrobial treatment, application method and day of display on ground beef redness (a*) during simulated retail display storage at $4^{\circ} \mathrm{C}$

\begin{tabular}{|c|c|c|c|c|c|c|c|}
\hline \multirow{2}{*}{ *Treatment } & \multirow{2}{*}{ **Application Method } & & \multicolumn{5}{|c|}{ Redness $\left(\mathbf{a}^{*}\right)$} \\
\hline & & & Day 0 & Day 1 & Day 2 & Day 3 & Day 7 \\
\hline $\mathrm{CON}$ & - & & $13.97^{\mathrm{c}}$ & 13.09 & $18.40^{\mathrm{abc}}$ & 20.30 & $20.21^{\mathrm{a}}$ \\
\hline PA & $\mathrm{CS}$ & & $15.59^{\mathrm{abc}}$ & 9.33 & $12.36^{\mathrm{abc}}$ & 17.78 & $18.16^{\mathrm{a}}$ \\
\hline W & $\mathrm{CS}$ & & $15.75^{\mathrm{abc}}$ & 11.17 & $18.68^{\mathrm{ab}}$ & 19.93 & $17.92^{\mathrm{ab}}$ \\
\hline W & ES & & $17.18^{\mathrm{abc}}$ & 12.16 & $19.97^{\mathrm{a}}$ & 19.60 & $17.81^{\mathrm{ab}}$ \\
\hline PM & $\mathrm{CS}$ & & $16.09^{\mathrm{abc}}$ & 8.95 & $10.12^{\mathrm{bc}}$ & 17.02 & $18.04^{\mathrm{ab}}$ \\
\hline PM & ES & & $17.19^{\mathrm{abc}}$ & 8.71 & $15.90^{\mathrm{abc}}$ & 16.12 & $14.62^{\mathrm{b}}$ \\
\hline $\mathrm{PP}$ & $\mathrm{CS}$ & & $14.56^{\mathrm{bc}}$ & 9.85 & $9.37^{\mathrm{c}}$ & 14.29 & $17.99^{\mathrm{ab}}$ \\
\hline PP & ES & & $18.06^{\mathrm{ab}}$ & 8.97 & $15.04^{\mathrm{abc}}$ & 19.21 & $18.02^{\mathrm{ab}}$ \\
\hline $\mathrm{PO}$ & $\mathrm{CS}$ & & $16.13^{\mathrm{abc}}$ & 9.45 & $16.93^{\mathrm{abc}}$ & 19.49 & $19.80^{\mathrm{ab}}$ \\
\hline $\mathrm{PO}$ & ES & & $15.12^{\mathrm{abc}}$ & 10.08 & $17.43^{\mathrm{abc}}$ & 18.80 & $18.65^{\mathrm{ab}}$ \\
\hline $\mathrm{PF}$ & $\mathrm{CS}$ & & $17.89^{\mathrm{abc}}$ & 9.59 & $15.82^{\mathrm{abc}}$ & 20.04 & $19.97^{\mathrm{ab}}$ \\
\hline \multirow[t]{2}{*}{$\mathrm{PF}$} & ES & & $18.98^{\mathrm{a}}$ & 9.56 & $14.10^{\mathrm{abc}}$ & 17.93 & $18.85^{\mathrm{ab}}$ \\
\hline & & Standard error & \pm 0.70 & \pm 0.86 & \pm 1.63 & \pm 1.43 & \pm 0.97 \\
\hline
\end{tabular}

Redness ( $\left.a^{*}\right)$ reported as least squares means along with \pm standard error.

${ }^{\mathrm{a}-\mathrm{c}}$ Least squares means within a column with different superscripts differed significantly $(P<0.05)$.

Treatments: $\mathrm{CON}=$ untreated inoculated control, $\mathrm{PA}=0.02 \%$ peroxyacetic acid; $\mathrm{W}=$ deionized water, $\mathrm{PM}=0.02 \%$ peroxyacetic acid followed by $3 \%$ malic acid, $\mathrm{PP}=0.02 \%$ peroxyacetic acid followed by $3 \%$ pyruvic acid, $\mathrm{PO}=0.02 \%$ peroxyacetic acid followed by $3 \%$ octanoic acid, $\mathrm{PF}=$ $0.02 \%$ peroxyacetic acid followed by saturated solution of fumaric acid.

**Application methods: CS = conventional spray application, ES = electrostatic spray application. 
Table 2.3 Effects of antimicrobial treatment, application method and day of display on ground beef yellowness $\left(b^{*}\right)$ during simulated retail display storage at $4^{\circ} \mathrm{C}$

\begin{tabular}{|c|c|c|c|c|c|c|c|}
\hline \multirow{2}{*}{ *Treatment } & \multirow{2}{*}{ **Application Method } & & \multicolumn{5}{|c|}{ Yellowness (b*) } \\
\hline & & & Day 0 & Day 1 & Day 2 & Day 3 & Day 7 \\
\hline $\mathrm{CON}$ & - & & 16.34 & 15.36 & 15.03 & 15.37 & 14.27 \\
\hline PA & $\mathrm{CS}$ & & 16.88 & 16.40 & 15.06 & 16.19 & 14.25 \\
\hline $\mathrm{W}$ & $\mathrm{CS}$ & & 16.87 & 16.08 & 17.36 & 16.57 & 13.64 \\
\hline W & ES & & 16.76 & 15.60 & 16.55 & 15.17 & 12.51 \\
\hline PM & $\mathrm{CS}$ & & 17.25 & 15.89 & 16.73 & 17.55 & 14.89 \\
\hline PM & ES & & 17.31 & 15.50 & 14.97 & 15.09 & 12.41 \\
\hline PP & $\mathrm{CS}$ & & 17.39 & 17.24 & 16.77 & 17.42 & 15.21 \\
\hline $\mathrm{PP}$ & ES & & 17.31 & 15.97 & 16.42 & 16.80 & 13.88 \\
\hline $\mathrm{PO}$ & $\mathrm{CS}$ & & 18.00 & 16.55 & 17.31 & 17.62 & 15.73 \\
\hline $\mathrm{PO}$ & ES & & 16.52 & 15.76 & 16.56 & 17.23 & 15.15 \\
\hline $\mathrm{PF}$ & $\mathrm{CS}$ & & 18.11 & 16.77 & 16.99 & 17.59 & 14.95 \\
\hline \multirow[t]{2}{*}{ PF } & ES & & 18.55 & 16.14 & 16.41 & 15.89 & 14.35 \\
\hline & & Standard error & 0.50 & 0.49 & 0.87 & 0.45 & 0.66 \\
\hline
\end{tabular}

Yellowness $\left(b^{*}\right)$ reported as least squares means along with \pm standard error, Least squares means did not differ $(P>0.05)$.

*Treatments: $\mathrm{CON}=$ untreated inoculated control, $\mathrm{PA}=0.02 \%$ peroxyacetic acid; $\mathrm{W}=$ deionized water, $\mathrm{PM}=0.02 \%$ peroxyacetic acid followed by $3 \%$ malic acid, $\mathrm{PP}=0.02 \%$ peroxyacetic acid followed by $3 \%$ pyruvic acid, $\mathrm{PO}=0.02 \%$ peroxyacetic acid followed by $3 \%$ octanoic acid, $\mathrm{PF}=$ $0.02 \%$ peroxyacetic acid followed by saturated solution of fumaric acid.

**Application methods: $\mathrm{CS}=$ conventional spray application, ES = electrostatic spray application.

The hue angles of ground beef from all treatments showed no difference $(P>$ 0.05 ) on days 0 and 7 of display (Table 2.4). On day 1 of display, only W treatment by both application methods and PO by ES method showed a similar hue angle $(P>0.05)$ to the control sample. All the treatments except CS application of PP, irrespective of application method, showed similar hue angle $(P>0.05)$ to the control ground beef on days 2 and 3 of display. All treated samples possessed a saturation index similar $(P>0.05)$ to control throughout the display time except ES applications of PF on day 0 of display (Table 2.5).
Therefore, with the exception of PF applied by ES on day 0 of display, all treatments were as vivid in color throughout display as the control. Additionally, all treatments had similar reflectance ratio (estimated oxymyoglobin content) compared to CON on days 0,3 and 7 of display (Table 2.6). Hence, antimicrobial or application method had little impact on myoglobin state, keeping similar oxymyoglobin content as the control.

Table 2.4 Effects of antimicrobial treatment, application method and day of display on ground beef hue angle during simulated retail display storage at $4^{\circ} \mathrm{C}$

\begin{tabular}{|c|c|c|c|c|c|c|c|}
\hline \multirow{2}{*}{ *Treatment } & \multirow{2}{*}{ **Application Method } & & \multicolumn{5}{|c|}{ Hue angle } \\
\hline & & & Day 0 & Day 1 & Day 2 & Day 3 & Day 7 \\
\hline $\mathrm{CON}$ & - & & 49.48 & $49.56^{\mathrm{b}}$ & $39.32^{\mathrm{c}}$ & $37.19^{\mathrm{b}}$ & 35.27 \\
\hline PA & CS & & 47.27 & $60.33^{\mathrm{a}}$ & $50.71^{\mathrm{abc}}$ & $42.33^{\mathrm{ab}}$ & 37.98 \\
\hline W & $\mathrm{CS}$ & & 46.97 & $55.45^{\mathrm{ab}}$ & $42.95^{\mathrm{c}}$ & $39.73^{\mathrm{b}}$ & 37.33 \\
\hline W & ES & & 44.26 & $52.36^{\mathrm{ab}}$ & $39.72^{\mathrm{c}}$ & $37.84^{\mathrm{b}}$ & 35.11 \\
\hline PM & $\mathrm{CS}$ & & 47.07 & $60.65^{\mathrm{a}}$ & $58.81^{\mathrm{abc}}$ & $45.99^{\mathrm{ab}}$ & 39.57 \\
\hline PM & ES & & 45.18 & $60.65^{\mathrm{a}}$ & $43.24^{\mathrm{c}}$ & $43.17^{\mathrm{ab}}$ & 40.35 \\
\hline PP & $\mathrm{CS}$ & & 50.09 & $60.23^{\mathrm{a}}$ & $60.79^{\mathrm{a}}$ & $51.39^{\mathrm{a}}$ & 40.14 \\
\hline PP & ES & & 43.81 & $60.67^{\mathrm{a}}$ & $47.63^{\mathrm{bc}}$ & $41.17^{\mathrm{ab}}$ & 37.61 \\
\hline $\mathrm{PO}$ & $\mathrm{CS}$ & & 48.14 & $60.25^{\mathrm{a}}$ & $46.07^{\mathrm{c}}$ & $42.09^{\mathrm{ab}}$ & 38.45 \\
\hline PO & ES & & 47.50 & $57.47^{\mathrm{ab}}$ & $43.52^{c}$ & $42.51^{\mathrm{ab}}$ & 39.08 \\
\hline $\mathrm{PF}$ & $\mathrm{CS}$ & & 45.37 & $60.26^{\mathrm{a}}$ & $47.50^{\mathrm{bc}}$ & $41.29^{\mathrm{ab}}$ & 36.79 \\
\hline \multirow[t]{2}{*}{ PF } & ES & & 44.35 & $59.33^{\mathrm{a}}$ & $49.44^{\mathrm{abc}}$ & $41.55^{\mathrm{ab}}$ & 37.30 \\
\hline & & $\begin{array}{l}\text { Standard } \\
\text { error }\end{array}$ & 1.17 & 1.62 & 2.09 & 1.99 & 1.12 \\
\hline
\end{tabular}

Hue angle $\left[\tan ^{-1}\left(\mathrm{~b}^{*} / \mathrm{a}^{*}\right)\right]$ reported as least squares means along with \pm standard error.

${ }^{\mathrm{a}-\mathrm{c}}$ Least squares means within a column with different superscripts differed significantly $(P<0.05)$.

*Treatments: $\mathrm{CON}=$ untreated inoculated control, $\mathrm{PA}=0.02 \%$ peroxyacetic acid; $\mathrm{W}=$ deionized water, $\mathrm{PM}=0.02 \%$ peroxyacetic acid followed by

$3 \%$ malic acid, $\mathrm{PP}=0.02 \%$ peroxyacetic acid followed by $3 \%$ pyruvic acid, $\mathrm{PO}=0.02 \%$ peroxyacetic acid followed by $3 \%$ octanoic acid, $\mathrm{PF}=$ $0.02 \%$ peroxyacetic acid followed by saturated solution of fumaric acid.

**Application methods: $\mathrm{CS}=$ conventional spray application, $\mathrm{ES}=$ electrostatic spray application. 
Table 2.5 Effects of antimicrobial treatment, application method and day of display on ground beef saturation index during simulated retail display storage at $4^{\circ} \mathrm{C}$

\begin{tabular}{|c|c|c|c|c|c|c|c|}
\hline \multirow{2}{*}{ *Treatment } & \multirow{2}{*}{ **Application Method } & & \multicolumn{5}{|c|}{ Saturation index } \\
\hline & & & Day 0 & Day 1 & Day 2 & Day 3 & Day 7 \\
\hline $\mathrm{CON}$ & - & & $21.51^{b}$ & 20.21 & 23.77 & 25.48 & $24.76^{\mathrm{ab}}$ \\
\hline PA & $\mathrm{CS}$ & & $23.00^{\mathrm{ab}}$ & 18.88 & 19.51 & 24.06 & $23.14^{\mathrm{ab}}$ \\
\hline $\mathrm{W}$ & $\mathrm{CS}$ & & $23.09^{\mathrm{ab}}$ & 19.61 & 25.52 & 25.92 & $22.53^{\mathrm{ab}}$ \\
\hline W & ES & & $24.02^{\mathrm{ab}}$ & 19.82 & 25.94 & 24.79 & $21.77^{\mathrm{ab}}$ \\
\hline PM & $\mathrm{CS}$ & & $23.60^{\mathrm{ab}}$ & 18.24 & 19.56 & 24.48 & $23.40^{\mathrm{ab}}$ \\
\hline PM & ES & & $24.40^{\mathrm{ab}}$ & 17.78 & 21.84 & 22.09 & $19.18^{\mathrm{b}}$ \\
\hline PP & CS & & $22.68^{\mathrm{ab}}$ & 19.86 & 19.21 & 22.65 & $23.56^{\mathrm{ab}}$ \\
\hline PP & ES & & $25.04^{\mathrm{ab}}$ & 18.32 & 22.28 & 25.56 & $22.75^{\mathrm{ab}}$ \\
\hline $\mathrm{PO}$ & $\mathrm{CS}$ & & $24.18^{\mathrm{ab}}$ & 19.06 & 24.27 & 26.29 & $25.29^{\mathrm{a}}$ \\
\hline $\mathrm{PO}$ & ES & & $22.41^{\mathrm{b}}$ & 18.72 & 24.05 & 25.51 & $24.03^{\mathrm{ab}}$ \\
\hline $\mathrm{PF}$ & CS & & $25.46^{\mathrm{ab}}$ & 19.31 & 23.30 & 26.68 & $24.95^{\mathrm{ab}}$ \\
\hline \multirow[t]{2}{*}{ PF } & ES & & $26.55^{\mathrm{a}}$ & 18.76 & 21.65 & 23.97 & $23.70^{\mathrm{ab}}$ \\
\hline & & $\begin{array}{l}\text { Standard } \\
\text { error }\end{array}$ & 0.72 & 0.82 & 1.64 & 1.01 & 1.08 \\
\hline
\end{tabular}

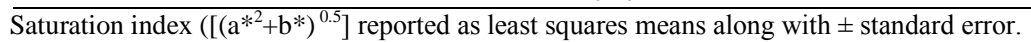

${ }^{\mathrm{a}-\mathrm{b}}$ Least squares means within a column with different superscripts differed significantly $(P<0.05)$.

*Treatments: $\mathrm{CON}=$ untreated inoculated control, $\mathrm{PA}=0.02 \%$ peroxyacetic acid; $\mathrm{W}=$ deionized water, $\mathrm{PM}=0.02 \%$ peroxyacetic acid followed by $3 \%$ malic acid, $\mathrm{PP}=0.02 \%$ peroxyacetic acid followed by $3 \%$ pyruvic acid, $\mathrm{PO}=0.02 \%$ peroxyacetic acid followed by $3 \%$ octanoic acid, $\mathrm{PF}=$ $0.02 \%$ peroxyacetic acid followed by saturated solution of fumaric acid. $* * * *$ Application methods: CS $=$ conventional spray application, ES $=$ electrostatic spray application.

Table 2.6 Effects of antimicrobial treatment, application method and day of display on ground beef reflectance ratio during simulated retail display storage at $4{ }^{\circ} \mathrm{C}$

\begin{tabular}{|c|c|c|c|c|c|c|c|}
\hline \multirow{2}{*}{ *Treatment } & \multirow{2}{*}{ **Application Method } & & \multicolumn{5}{|c|}{ Reflectance ratio } \\
\hline & & & Day 0 & Day 1 & Day 2 & Day 3 & Day 7 \\
\hline $\mathrm{CON}$ & - & & $2.82^{\mathrm{abc}}$ & $1.38^{\mathrm{a}}$ & $2.20^{\mathrm{ab}}$ & 2.53 & 2.37 \\
\hline PA & $\mathrm{CS}$ & & $2.83^{\mathrm{abc}}$ & $1.04^{\mathrm{b}}$ & $1.45^{\mathrm{bcd}}$ & 2.21 & 2.59 \\
\hline W & $\mathrm{CS}$ & & $2.86^{\mathrm{ab}}$ & $1.21^{\mathrm{ab}}$ & $2.12^{\mathrm{ab}}$ & 2.46 & 2.36 \\
\hline W & ES & & $3.18^{\mathrm{a}}$ & $1.29^{\mathrm{ab}}$ & $2.41^{\mathrm{a}}$ & 2.55 & 2.68 \\
\hline PM & $\mathrm{CS}$ & & $2.84^{\mathrm{abc}}$ & $1.05^{\mathrm{ab}}$ & $1.11^{\mathrm{d}}$ & 2.08 & 2.38 \\
\hline PM & ES & & $2.92^{\mathrm{ab}}$ & $1.05^{\mathrm{ab}}$ & $1.89^{\mathrm{abc}}$ & 2.19 & 2.13 \\
\hline PP & CS & & $2.58^{\mathrm{bc}}$ & $1.09^{\mathrm{ab}}$ & $1.29^{\mathrm{cd}}$ & 1.81 & 2.37 \\
\hline PP & ES & & $3.14^{\mathrm{ab}}$ & $1.07^{\mathrm{ab}}$ & $1.86^{\mathrm{abcd}}$ & 2.44 & 2.48 \\
\hline PO & $\mathrm{CS}$ & & $2.78^{\mathrm{abc}}$ & $1.07^{\mathrm{ab}}$ & $1.97^{\mathrm{abc}}$ & 2.33 & 2.35 \\
\hline $\mathrm{PO}$ & ES & & $2.28^{\mathrm{c}}$ & $1.34^{\mathrm{ab}}$ & $1.96^{\mathrm{abc}}$ & 2.33 & 2.68 \\
\hline $\mathrm{PF}$ & $\mathrm{CS}$ & & $3.17^{\mathrm{a}}$ & $1.02^{\mathrm{b}}$ & $1.77^{\mathrm{abcd}}$ & 2.33 & 2.42 \\
\hline \multirow[t]{2}{*}{$\mathrm{PF}$} & ES & & $3.34^{\mathrm{a}}$ & $1.06^{\mathrm{ab}}$ & $1.65^{\mathrm{bcd}}$ & 2.26 & 2.21 \\
\hline & & Standard error & 0.10 & 0.06 & 1.33 & 0.18 & 0.22 \\
\hline
\end{tabular}

Reflectance ratio $(580 / 630 \mathrm{~nm})$ reported as least squares means along with \pm standard error,

${ }^{\mathrm{a}-\mathrm{c}}$ Least squares means within a column with different superscripts differed significantly $(P<0.05)$

*Treatments: $\mathrm{CON}=$ untreated inoculated control, $\mathrm{PA}=0.02 \%$ peroxyacetic acid; $\mathrm{W}=$ deionized water, $\mathrm{PM}=0.02 \%$ peroxyacetic acid followed by $3 \%$ malic acid, $\mathrm{PP}=0.02 \%$ peroxyacetic acid followed by $3 \%$ pyruvic acid, $\mathrm{PO}=0.02 \%$ peroxyacetic acid followed by $3 \%$ octanoic acid, $\mathrm{PF}=$ $0.02 \%$ peroxyacetic acid followed by saturated solution of fumaric acid.

**Application methods: CS = conventional spray application, ES = electrostatic spray application.

A limited number of studies have evaluated the effects of malic, octanoic, pyruvic and fumaric acid applied as a multiple hurdle intervention on beef trimmings in decreasing E. coli $(\mathrm{O} 157: \mathrm{H} 7, \mathrm{O} 26, \mathrm{O} 103, \mathrm{O} 111, \mathrm{O} 121, \mathrm{O} 45$ and O145) and Salmonella (S. Typhimurium DT 104, and S. Newport) populations on resultant ground beef. According to Chen et al., (2012), antimicrobial properties of peroxyacetic acid are attributed to its high oxidation potential. Studies related to peroxyacetic acid as a decontaminant supported the efficacy of peroxyacetic acid to reduce a variety of microorganisms in beef products (Ellebracht et al., 2005; Gill and Badoni, 2004; Pohlman et al., 2009; Quilo et al., 2010; Vandekinderen, et al., 2009). Ellebracht et al. (2005) reported that $200 \mathrm{ppm}$ peroxyacetic acid was able to reduce rifampicin-resistant $E$. coli O157:H7 and S. Typhimurium in beef trims by approximately $1 \log$ CFU. A study by Quilo et al. (2010) also reported application of peroxyacetic acid followed by potassium lactate treatment on beef trimmings by vacuum tumbling method could effectively reduce Escherichia coli and nalidixic acid resistant Salmonella Typhimurium in resulting ground beef. Also based on Kalchayanand et al. (2012), application of peroxyacetic acid at $200 \mathrm{ppm}$ on inoculated beef flanks could be an effective intervention against the top six non-O157 organisms. In agreement, our results signified the competence of peroxyacetic acid with $1.4 \mathrm{log}$ reduction in cumulative populations of $E$. coli $\mathrm{O} 157: \mathrm{H} 7$ and six serogroups of non-O157:H7 and 1.7 log Salmonella population (Salmonella Typhimurium DT 104 and Salmonella Newport MDR-AmpC) when applied as a single intervention on beef trimmings. Additionally, even though application of multiple chemicals expected to enhance the microbial reduction, peroxyacetic acid alone achieved lower reduction in E. coli and Salmonella populations on day 0 of display than did multiple treatment combinations. However, this trend was changed on day 1 of display and some multiple treatment combinations prompted higher effectiveness in reducing E. coli and Salmonella populations. This change may be attributed to the residual bactericidal effect of organic acid caused by $\mathrm{pH}$ decrease within bacterial cell (Malicki et al., 2004). As Dibner et al. (2002) specified the antimicrobial effect of the organic acids primarily caused by nondissociated form of organic acid. Once diffused into bacterial cells, nondissociated organic acids will dissociate into anions and protons which force an inhibitory effect on microorganisms. Because the excretion of protons from the cell is energy consuming process, it will eventually deplete bacterial metabolism leading the cells to death. In addition the anions also can interfere with processes in the cytoplasm. According to Skrivanova et al. (2006), the structure of organic acids may play a role in diffusion into the cell and thus acids with a shorter chain may have a stronger lethal effect. Meat $\mathrm{pH}$ is one of the major factors that determines microorganism growth and most organisms grow well at $\mathrm{pH}$ value of 7 (Jay et al., 2005). Although, reduction in microbial populations for all treatments on day $0-3$ of display of our study might be the result of lowered initial surface $\mathrm{pH}$, less reduction of microbial populations on day 7 might be attributed to the buffering of $\mathrm{pH}$ values of the treated and untreated ground beef samples, pushing the $\mathrm{pH}$ higher over display time (Figure 1). 


\section{Electrostatic spray (ES)}

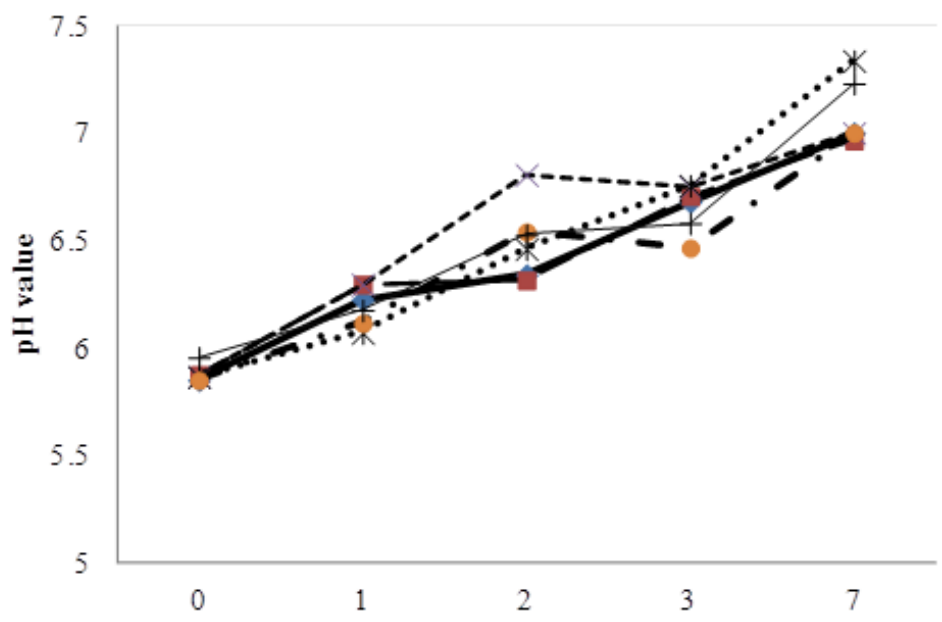

Connventional spray (CS)

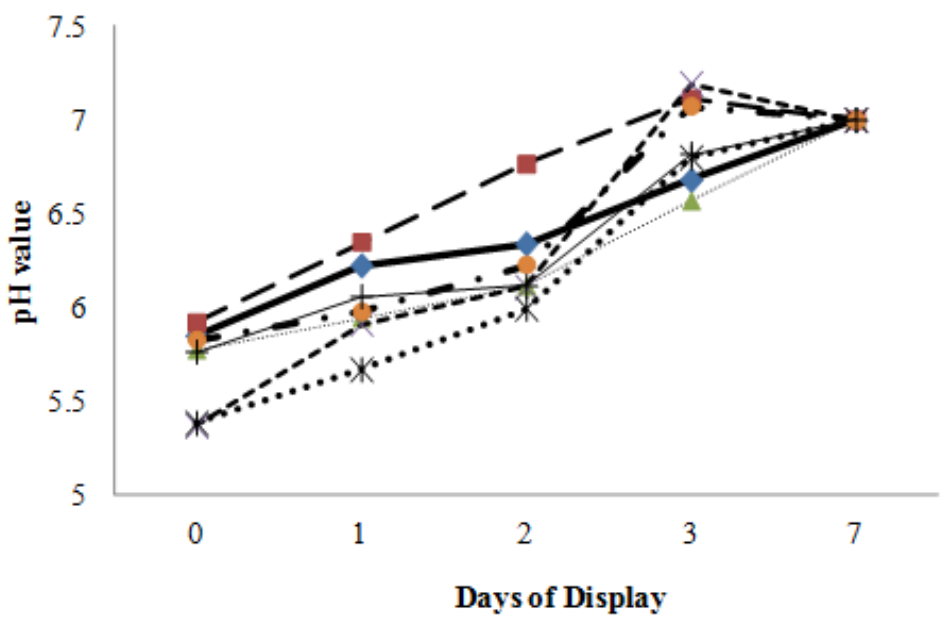

Figure 1 Ground beef $\mathrm{pH}$ values with different treatments and spray application methods

Legend:

$\longrightarrow \mathrm{CON}$

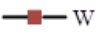

$--\div-\cdot P M$

$\cdots *$ PP $\quad-\cdot \cdot \mathrm{PO}$

Treatments: $\mathrm{CON}=$ untreated inoculated control, $\mathrm{PA}=0.02 \%$ peroxyacetic acid $\mathrm{W}=$ deionized water, $\mathrm{PM}=0.02 \%$ peroxyacetic acid followed by $3 \%$ malic acid $\mathrm{PP}=0.02 \%$ peroxyacetic acid followed by $3 \%$ pyruvic acid, $\mathrm{PO}=0.02 \%$ peroxyacetic acid followed by $3 \%$ octanoic acid, $\mathrm{PF}=0.02 \%$ peroxyacetic acid followed by saturated solution of fumaric acid.

**Application methods: CS = conventional spray application, $\mathrm{ES}=$ electrostatic spray application.

Fumaric acid is a dicarboxylic acid and a very important acidulent agent and an antifungicide in the food industry (Dang et al., 2009). Among all the organic acids tested, fumaric acid had very poor water solubility. However, it showed a comparable antimicrobial effect to other organic acids tested. As stated by Lilard (1988), bacteria became entrapped in crevices on tissue surfaces, such as beef trimmings, may impair antimicrobial efficiency. Therefore, efficient treatment application methods are important in enhancing antimicrobial performances Utilizing a liquid electrostatic coating system is quite feasible for decontamination applications in beef plants and has the ability to produce very even liquid electrostatic coatings efficiently (Abu-Ali and Barringer 2005). A study by Ganesh et al. (2010) reported 2\% malic acid in combination with $3 \%$ Grape Seed Extract (GSE) or lactic acid applied by electrostatic spray lowered $S$ Typhimuium counts on spinach by $2.6-3.3 \log$ CFU and produced higher inhibition of pathogens than conventional spray applications. Our results also showed electrostatic application of some organic acids may have similar or greater efficiency in controlling ground beef microbial populations compared to the conventional spray application of the same acid.

Even though it is assumed that organic acids potentially could accelerate the oxidation of myoglobin causing undesirable quality attributes, previous research recognize the extent of treatment variability is responsible for such changes
(Smulder and Geer, 1998). Spray application of 1 or $2 \%$ acetic or lactic plus mixture on beef steaks (Dixon et al., 1987) or beef strip loins (Acuff $\boldsymbol{e t} \boldsymbol{a l}$. 1987) showed no effect in meat color whereas dip application of $2 \%$ lactic or acetic on beef steaks showed discoloration (Kotula and Thelappurate, 1994) As reported by Quilo et al., (2009a, 2009b), the application of $0.02 \%$ peroxyacetic as a single antimicrobial intervention on beef trimmings prior to grinding had the ability to improve redness in resulting bulk ground beef or patties. Correspondingly, our treated samples, on most occasions, showed similar or enhanced redness to the untreated control ground beef. The effectiveness of octanoic acid based treatment as post-leathality interventions to reduce $L$. monocytgenes on ready- to- eat (RTE) meat and poultry products was reported by Burnett et al. (2007). Additionally, these treatments demonstrated minimum impact on sensory quality.

\section{CONCLUSION}

Peroxyacetic acid alone or followed by conventional or electrostatic spray application of malic, pyruvic, octonoic or fumaric acid on beef trimmings may be effective in reducing $E$. coli $\mathrm{O} 157: \mathrm{H} 7$ as well as non-STEC serotypes and Salmonella species through 2 days of display. These interventions had no or little interference on quality attributes of ground beef such as ground beef color. The ES application of organic acids established a cost-conscious treatment application with less antimicrobial usage as well as less waste management.

Therefore, the outcome of this study opens new avenues for cost-effective utilization of natural organic acids in more efficient decontamination interventions in ground beef production lines to reduce pathogens of recent concern.

Acknowledgements: The authors thank R. D. Guidry, C. L. Coffman, Brandon McCord, Sean McCord and Daniel McFarland for laboratory support.

\section{REFERENCES}

ABU-ALI, J., BARRINGER, S. A. 2008. Optimization of liquid electrostatic coating. Journal of Electrostatics, 66,184-189. http://dx.doi.org/10.1016/j.elstat.2007.12.003

ABU-ALI, J., BARRINGER, S. A. 2005. Method for electrostatic atomization in an EHD system. Journal of Electrostatics, 63, 361-369. http://dx.doi.org/10.1016/j.elstat.2004.11.004

ACUFF, G., VANDERZANT, J., SAVELL, D., JONES, D., GRIFFIN, J. EHLERS. 1987. Effect of acid decontamination of beef subprimal cuts on the microbiological and sensory characteristics of steaks. Meat Science, 19, 217-226 http://dx.doi.org/10.1016/0309-1740(87)90059-3

AYEMERICH, T., PICOUET, P. A., MONFORT, J. M. 2008. Decontamination technologies for meat products. Meat Science, 78,114-129. http://dx.doi.org/10.1016/j.meatsci.2007.07.007

BERRY, E. D., CUTTER, C. N. 2000. Effects of acid adaptation of Escherichia coli $\mathrm{O} 157: \mathrm{H} 7$ on efficacy of acetic acid spray washes to decontaminate beef carcass tissue. Applied Environmental Microbiology, 66, 1493-1498 http://dx.doi.org/10.1128/aem.66.4.1493-1498.2000

BRAKATE, M. L., ACUFF, G. R., LUCIA, L. M., HALE, D. S. 1993. Hot water decontamination of beef carcasses for reduction of initial bacterial numbers. Mea Science, 35, 397-401. http://dx.doi.org/10.1016/0309-1740(93)90044-i

CHEN, J. H., SEOW, R. J., LIU, T., YUK, H. G. 2012. Intervention Technologies for ensuring microbiological safety of meat: Current and future trend. Comprehensive Reviews in Food Science and Food Safety, 11, 199-132. http://dx.doi.org/10.1111/j.1541-4337.2011.00177.x

CUTTER, C. N., RIVERA-BETANCOURT, M. 2000. Interventions for the reduction of Salmonella Typhimurium DT 104 and Non-O157:H7 enterohemorrhagic Escherichia coli on beef surfaces. Journal of Food Protection, 63, 1326-1332.

DANG, L., W. DU, S. BLACK, and H. WEI. 2009. Solubility of Fumaric Acid in Propan-2-ol, Ethanol, Acetone, Propan-1-ol, and Water. Journal of Chemical and Engineering Data. 54, 3112-3113. DIBNER, J. J., BUTIN, P. 2002. Use of organic acids as a model to study the impact of gut microflora on nutrition and metabolism. Journal of Applied Poultry Research, 11, 453-63. http://dx.doi.org/10.1021/je9001637

DIBNER, J. J., BUTIN, P. 2002. Use of organic acids as a model to study the impact of gut microflora on nutrition and metabolism. Journal of Applied Poultry Research. 11:453-63. http://dx.doi.org/10.1093/japr/11.4.453

DICKSON, J. S., ANDERSON, M. E. 1992. Microbiological decontamination of animal carcasses by washing and sanitizing systems. A review. Journal of Food Protection, 55, 133-140.

DIXON, Z., VANDERZANT, C., ACUFF, G., SAVELL, J., JONES, D. 1987 Effect of acid treatment of beef strip loin steaks on microbiological and sensory characteristics. International Journal of Microbiology, 5, 181-186. http://dx.doi.org/10.1016/0168-1605(87)90035-3

DUBAL, Z. B., PATUKAR, A. M., WASKAR, V. S., ZENDE, R. J., LATHA C., RAWOOL, D. B., KADAM, M. M. 2004. Effect of food grade organic acids on inoculated $S$. aureus, L. monocytogenes, E. coli and $S$. Typhimurium in 
sheep/goat meat stored at refrigeration temperature. Meat Science, 66, 817-821. http://dx.doi.org/10.1016/j.meatsci.2003.08.004

EDWARDS, J. R., FUNG, D. Y. C. 2006. Prevention and decontamination of Escherichia coli $\mathrm{O} 157: \mathrm{H} 7$ on raw beef carcasses in commercial beef abattoirs. Journal of Rapid Methods and Automation in Microbiology, 14, 1-95. http://dx.doi.org/10.1111/j.1745-4581.2006.00037.x

ELLEBRACHT, J. W., KING, D. A., CASTILLO, A., LUCIA, L. M ACUFF, G. R., HARRIS, K. B., SAVELL, J. W. 2005. Evaluation of peroxyacetic acid as a potential pre-grinding treatment for control of Escherichia coli O 157:H7 and Salmonella Typhimurium on beef trimmings. Meat Science, 70, 197-203. http://dx.doi.org/10.1016/j.meatsci.2005.01.003

GANESH, V., HETTIARACHCHY, N. S., RAVICHANDRAN, M., JOHNSON, M. G., GRIFFIS, C. L., MARTIN, E. M., MEULLENET, J-F AND RICKE, S. C. 2010. Electrostatic spray of food-grade acids and plant extracts are more effective than conventional sprays in decontaminating Salmonella Typhimurium on Spinach. Journal of Food Science, 75, 391-396. http://dx.doi.org/10.1111/j.1750 3841.2010.01859.x

GILL, C. O., BADONI, M. 2004. Effects of peroxyacetic acid, acidified sodium chlorite or lactic acid solutions on the microflora of chilled beef carcasses International Journal of Food Microbiology, 91, 43-50. http://dx.doi.org/10.1016/s0168-1605(03)00329-5

GREER, G. G., DILTS, B. D. 1992. Factors affecting the susceptibility of meatborne pathogens and spoilage bacteria to organic acids. Food Research International, 25, 355-364. http://dx.doi.org/10.1016/0963-9969(92)90110-q HARDIN, M., ACUFF, G., LUCIA, L., OMAN, J., SAVELL, J. 1995. Comparison of methods for decontamination from beef carcasses. Journal of Food Protection, 58, 368-374.

HOGUE, A., AKKINA, J., ANGUlO, F., JOHNSON, R., PETERSON, K. AINI, P., SCHLOSSER, W. 1997. Situations assessment: Salmonella Typhimurium DT104. U. S. Department of Agriculture, Food Safety and Inspection Service;

http://www.aphis.usda.gov/animal health/emergingissues/downloads/dt104.pdf HUFFMAN, R. D. 2002. Current and future technologies for the decontamination of carcass and fresh meat. Meat Science, 62, 285-294 http://dx.doi.org/10.1016/s0309-1740(02)00120-1

JAY, J. M., LOESSNER, M. J., GOLDEN, D. A. (ed.). 2005. Modern Food Microbiology: Indicators of food microbial quality and safety. Springer Science and Business Media, New York. http://dx.doi.org/10.1007/0-387-23413-6 20 KALCHAYANAND, N., ARTHUR, T. M., BOSELEVAC, J. M., SCHMIDT, J. W., WANG, R., SHACKELFORD, S. D., WHEELER, T. L. 2012. Evaluation of commonly used antimicrobial interventions for fresh beef inoculated with shiga toxin producing Escherichia coli serotypes O26, O45, O103, O111, O121, O145 and O157:H7. Journal of Food Protection, 75, 1207-1212. http://dx.doi.org/10.4315/0362-028x.jfp-11-531

KING, D.A., LUCIA, L. M., CASTILlO, A. ACUFF, G. R., HARRIS, K. B. SAVELL, J. W. 2005. Evaluation of peroxyacetic acid as a post-chilling intervention for control of Escherichia coli 0157:H7 and Salmonella Typhimurium on beef carcass surfaces. Meat Science, 69, 401-407. http://dx.doi.org/10.1016/j.meatsci.2004.08.010

KOTULA, K., THELAPPURATE, R. 1994. Microbiological and sensory attributes of retail cuts of beef treated with acetic and lactic acid solutions. Journal of Food Protection, 57, 665-670.

LEVINE, A. S., FELLERS, C. R. 1940. Action of acetic acid on food spoilage microorganism. Journal of Bacteriology, 39, 499-515.

LILLARD, H. S. 1988. Effect of surfactant or changes in ionic strength on the attachment of Salmonella typhimurium to poultry skin and muscle. Journal of Food Science, 53, 727-730. http://dx.doi.org/10.1111/j.1365 2621.1988.tb08941.x

MALICKI, A., ZAWADZKI, W., BRUZEWICZ, S., GRACZYK, S., CZERSKI, A. 2004. Effect of formic and propionic acid mixture on Escherichia coli in fish meal stored at $12{ }^{\circ} \mathrm{C}$. Pakistan. Journal of Nutrition, 3, 353-356. http://dx.doi.org/10.3923/pjn.2004.353.356

MANI-LOPEZ, E., GRACIA, H. S., LOPEZ-MALO, A. 2011. Organic acids as antimicrobials to control Salmonella in meat and poultry products. Food Research International accessed at http://dx.doi:10.1016/J.foodres.2011.04.043

MEAD, P. S., FINELLI, L., SPITALNY, K. 1995. Risk factors for sporadic infection with Escherichia coli O157:H7. Abstract In: 44th Annual Epidemic Intelligence Service Conference, March 27-31, Atlanta, Georgia.

NATARO, J. P., KAPER, J. B. 1998. Diagnosis and investigation of diarrheagenic Escherichia coli. Clinical. Microbiology Review, 11, 142-201. http://dx.doi.org/10.1385/0-89603-498-4:387

POHLMAN, F. W., DIAS-MORSE, P. N., QUILO, S. A., BROWN, A. H. JR., CRANDALL, P. G., BAUBLITS, R. T., STORY, R. P. BOKINA, C., RAJARATNAM, G. 2009. Microbial, instrumental color and sensory characteristics of ground beef processed from beef trimmings treated with potasium lactate, sodium metasilicate, peroxyacetic acid, or acidified sodium chlorite as single antimicrobial interventiions. Journal of Muscle Foods, 20, 54 69. http://dx.doi.org/10.1111/j.1745-4573.2008.00133.x

POHLMAN, F. W., STIVARIUS, M. R., MCELYEA, K. S., JOHNSON, Z. B., JOHNSON, M. G. 2002a. The effects of ozone, chlorine dioxide, cetylpyridinium chloride and trisodium phosphate as multiple antimicrobial interventions on microbiological, instrumental color, sensory color, and odor characteristics of ground beef. Meat Science, 61, 307-313. http://dx.doi.org/10.1016/s0309 1740(01)00198-X

POHLMAN, F. W., STIVARIUS, M. R., MCELYEA, K. S., JOHNSON, Z. B., JOHNSON, M. G., 2002b. Reduction of microorganisms in ground beef using multiple intervention technology. Meat Science, 61, 315-322. http://dx.doi.org/10.1016/s0309-1740(01)00199-1

QUILO, S. A., POHLMAN, F. W., DIAS-MORSE, P. N., BROWN, A. H. JR. CRANDALL, P. G., STORY, R. P. 2010. Microbial, Instrumental color and sensory characteristics of inoculated ground beef produced using potassium lactate, sodium metasilicate, or peroxyacetic acid. Meat Science, 84, 470-476. http://dx.doi.org/10.1016/j.meatsci.2009.09.018

QUILO, S. A., POHLMAN, F. W., BROWN, A. H. JR., CRANDALL, P. G. DIAS-MORSE, P. N., BAUBLITS, R. T., APARICIO, J. L. 2009a. Effects of potassium lactate, sodium metasilicate, peroxyacetic acid, and acidified sodium chlorite on physical, chemical, and sensory properties. Meat Science, 82, 44-52. http://dx.doi.org/10.1016/j.meatsci.2008.12.002

QUILO, S. A., POHLMAN, F. W., DIAS-MORSE, P. N., BROWN, A. H. Jr., CRANDALL, P. G., BAUBLITS, R. T., APARICIO, J. L. 2009b. The impact of single antimicrobial intervention treatment with potassium lactate, sodium metasilicate, peroxyacetic acid, and acidified sodium chlorite, on non-inoculated ground beef lipid, instrumental color, and sensory characteristics. Meat Science, 83, 345-350. http://dx.doi.org/10.1016/j.meatsci.2009.05.015

RAMIREZ, A. J., ACUFF, G. R., LUCIA, L. M., SAVELL, J. W. 2001. Lactic acid and trisodium phosphate treatment of lamb breast to reduce bacterial contamination. Journal of Food Protection, 64, 1439-1441.

RANSOM, J. R., BELK, K. E., SOFOS, J. N., STOPFORTH, J. D., SCANGA, J. A., SMITH, G. C. 2003. Comparison of intervention technologies for reducing Escherichia coli $\mathrm{O} 157: \mathrm{H} 7$ on beef cuts and trimmings. Food Protection Trends, 1, 24-34.

RICKE, S. C. 2003. Perspectives on the use of organic acids and short chain fatty acids as antimicrobials. Poultry Science, 82, 632-639. http://dx.doi.org/10.1093/ps/82.4.632

ROLLER S. (ed.). 2003. Natural Antimicrobials for the minimal processing of foods: Organic acids. CRC Press, Boca Raton, FL. http://dx.doi.org/10.1533/9781855737037

SKRIVANOVA, E., MAROUNEK, M., BRENDA, V., BREZINA, P. 2006. Susceptibility of Escherichia coli, Salmonella sp. and Clostridium perfrigens to organic acid and monolaurin. Vetrinarni Medicina, 51, 81-88.

SMULDERS, F. J. M., GREER, G. G. 1998. Integrating microbia decontamination with organic acids in HACCP programs for muscle foods: prospects and controversies. J. Int. Food Microbiology, 44, 149-169. http://dx.doi.org/10.1016/s0168-1605(98)00123-8

SOFOS, J. N., G. C. SMITH. 1998. Nonacid meat decontamination technologies: Model studies and commercial applications. International Journal of Food Microbiology, 44, 171-188. http://dx.doi.org/10.1016/s0168-1605(98)00136-6

VANDEKINDEREN, I., DEVILEGHERE, F., DE MEULENAER, D. RAGAERT, P., VAN CAMP, J. 2009. Optimization and evaluation of a decontamination stepwith peroxyacetic acid for fresh-cut produce. Food Microbiology, 26, 882-888. http://dx.doi.org/10.1016/j.fm.2009.06.004 